\title{
Características epidemiológicas de la neumonía intrahospitalaria en un servicio de medicina interna del Hospital Guillermo Almenara Irigoyen de Lima, Perú
}

\author{
César León-Chahua', Teodoro Oscanoa-Espinoza², Cynthia Chávez-Gutiérrez ${ }^{3}$, José Chávez-Gutiérrez ${ }^{4}$
}

RESUMEN

Objetivo: Determinar las características epidemiológicas de los pacientes con neumonía intrahospitalaria $(\mathrm{NIH})$ en un servicio de medicina interna.

Material y métodos: Estudio descriptivo, retrospectivo; llevado a cabo en el servicio de medicina interna $\mathrm{N}^{\circ} 5$ del Hospital Nacional Guillermo Almenara Irigoyen (HNGAI) durante el año 2015. Se revisaron las historias clínicas de pacientes mayores de 15 años con el diagnóstico de egreso de NIH que cumplieron los criterios de selección.

Resultados: Se evaluaron 26 historias clínicas de pacientes. La incidencia de NIH fue de $2.37 \%$ y la mortalidad de $73.03 \%$. La edad promedio fue de $58.69 \pm 12.49$ años. El $42.31 \%$ de pacientes tuvieron más de 80 años. La NIH fue predominante en el género masculino $65.38 \%$. Se obtuvo una estancia hospitalaria promedio de 62.53 días y 12 pacientes $46.15 \%$ estuvieron más de 60 días hospitalizados. Entre los factores intrínsecos asociados, la enfermedad cerebrovascular fue la comorbilidad más frecuente con $26.92 \%$; seguido de la enfermedad neoplásica con $23.07 \%$. Los factores extrínsecos asociados más frecuentes fueron el uso de sonda nasogástrica y la profilaxis de úlceras de stress con $84.61 \%$ cada uno, la intubación endotraqueal $53.84 \%$ y la ventilación mecánica $50 \%$. Los microorganismos aislados fueron Acinetobacter baumannii $15.38 \%$, Pseudomona aeruginosa $11.53 \%$ y Staphilococcus aureus $(3.84 \%)$.

Conclusiones: Se encontró una alta mortalidad en los pacientes con NIH en el servicio de medicina interna. La edad avanzada, las comorbilidades tales como enfermedad cerebrovascular, la enfermedad neoplásica, el uso de sonda nasogástrica y de inhibidores de la secreción gástrica son factores mayormente encontrados en NIH.

Palabras clave: neumonía intrahospitalaria, características epidemiológicas, factores asociados, estancia hospitalaria.

\section{Epidemiological features of Hospital-acquired Pneumonia in an Internal Medicine Service from Guillermo Almenara Irigoyen Hospital in Lima, Peru}

\section{ABSTRACT}

Objetive: To determine the epidemiological features in patients with hospital-acquired pneumonia (HAP) in an Internal Medicine Service.

Material and methods: A retrospective and descriptive study was carried out in Internal Medicine Service $\mathrm{N}^{\circ} 5$ from Guillermo Almenara Irigoyen National Hospital in 2015. Medical records of patients older than 15 years old were reviewed with the discharged diagnosis of HAP who meet the selection criteria.

Results: Twenty six medical records were evaluated. The incidence rate of HAP was $2.37 \%$ and the mortality rate was $73.03 \%$. The average age was $58.69 \pm 12.49$ years old. Patients older than 80 years old meant $42.31 \%$ of all of them. HAP was more frequent in male patients (65.38\%). The average hospital stay was 62.53 days and 12 patients $(46.15 \%)$ had more than sixty days of hospitalization. Among the intrinsic associated factors, the cerebrovascular disease was the most frequent illness, followed by the neoplastic disease with $23.07 \%$. The most frequent extrinsic associated factors were the use of nasogastric tube and the stress ulcers prophylaxis with $84.61 \%$ each other, the endotracheal intubation with $53.84 \%$ and the mechanical ventilation with 50\%. The isolated microorganisms were Acinetobacter baumannii (15.38\%), Pseudomona aeruginosa $(11.53 \%)$ and Staphilococcus aureus (3.84\%).

Conclusions: A high mortality rate was found in patients with HAP. Advanced age, comorbidities like cerebrovascular and neoplastic disease and the use of nasogastric tube and gastric secretor inhibitors were factors mostly found in HAP.

Key words: hospital-acquired pneumonia, epidemiological features, associated factors, hospital stay.

1. Servicio de Medicina Interna, Hospital Nacional Guillermo Almenara Irigoyen-EsSalud, Lima.

2. Departamento de Medicina Interna, Hospital Guillermo Almenara Irigoyen-EsSalud, Lima.

3. Facultad de Medicina, Universidad San Martín de Porres, Lima.

4. Facultad de Medicina, Universidad San Martín de Porres, Lima. 


\section{INTRODUCCIÓN}

Las infecciones intrahospitalarias son procesos no esperados que se presentan en las diferentes áreas de atención hospitalaria, convirtiéndose en un desafío para la comunidad médica. Es en este contexto que la NIH se constituye como la infección intrahospitalaria más frecuente en unidad de cuidados intensivos ( $\mathrm{UCI}$ ) y la segunda en frecuencia luego de la infección urinaria en áreas de hospitalización, de allí el interés creciente por esta afección ${ }^{(1,2)}$.

La NIH es la infección del parénquima pulmonar que se desarrolla luego de más de 48 horas y no estuvo incubando antes de su ingreso ${ }^{(2,3)}$. En su clasificación actual se incluye la neumonía adquirida en el hospital, la neumonía asociada a ventilación mecánica y la neumonía asociada a cuidados de salud. Se puede hacer una división por el tiempo de aparición de la neumonía en NIH de inicio temprano hasta los primeros cuatro días de hospitalización con gérmenes de origen comunitario y en $\mathrm{NIH}$ de inicio tardío a partir del quinto día, con patógenos hospitalarios que colonizan la orofaringe ${ }^{(4,5)}$.

En relación a la incidencia, se estima en 5 a 10 casos por 1000 pacientes hospitalizados. La edad es un factor influyente en la frecuencia de la $\mathrm{NIH}$. En menores de 35 años de edad esta es de 5 casos por 1000 , y en los mayores de 65 años esta se eleva a más de 15 casos por 1000 pacientes hospitalizados ${ }^{(4)}$. En los pacientes que ingresan a ventilación mecánica, la incidencia se multiplica hasta por veinte ${ }^{(2,4)}$.

La microaspiración es el mecanismo de transporte más frecuente por el cual las secreciones contaminadas llegan al tejido pulmonar desde la mucosa orofaríngea, placa dental, senos paranasales $y$, en menor medida, el tracto gastrointestinal, lo que da como resultado el posterior desarrollo de NIH.

Para el diagnóstico de NIH se tienen en cuenta hallazgos clínicos, resultados de laboratorio, de radiología y microbiológicos. En el año 2005, la Sociedad Americana de Tórax (SAT) propone uniformizar el diagnóstico y define la presencia de $\mathrm{NIH}$ en el paciente que presenta un infiltrado radiológico pulmonar nuevo o una progresión del infiltrado pulmonar ya existente, más dos de tres criterios clínicos como leucocitosis o leucopenia, fiebre mayor de $38^{\circ} \mathrm{C}$ y presencia de secreciones bronquiales purulentas ${ }^{(4)}$.

El aislamiento del agente causal puede realizarse a partir de muestras obtenidas del árbol bronquial por procedimientos invasivos o no invasivos. La literatura reporta que los microorganismos aislados con mayor frecuencia son gram negativos intrahospitalarios con alta resistencia antibiótica. Weyland y col. ${ }^{(6)}$ realizaron un estudio en la $\mathrm{UCl}$ y reportaron como los gérmenes más frecuentes a Acinetobacter baumannii, Staphylococcus aureus y Pseudomona aeruginosa con un $37.9 \%, 21.3 \%$ y $20.9 \%$ respectivamente. En otro estudio, Magret y col. ${ }^{(7)}$ en resultados de hemocultivo de pacientes con NIH en $\mathrm{UCl}$ aislaron Staphilococcus aureus con 22.6\%, Acinetobacter baumanii con $17.9 \%$ y Pseudomona aeruginosa con $14.3 \%$

Se ha llegado a determinar que existen condiciones inherentes al huésped y factores externos que influyen para el desarrollo de la $\mathrm{NIH}$, estas han sido divididas en factores intrínsecos y factores extrínsecos. Los factores de riesgo intrínsecos son los que disminuyen las defensas del huésped y lo hacen más vulnerable a procesos infecciosos tales como enfermedades crónicas, entre ellas la diabetes mellitus ${ }^{(8,9,10)}$, enfermedad pulmonar obstructiva crónica (EPOC), enfermedad renal crónica, diálisis, enfermedad neurológica con deterioro de conciencia ${ }^{(11,12)}$, enfermedad neoplásica y la edad del paciente. Los factores de riesgo extrínsecos son los procedimientos diagnósticos y terapéuticos en los que se incluyen la intubación endotraqueal, ingreso a ventilación mecánica, la colocación de sonda nasogástrica ${ }^{(13)}$, aspiración de secreciones y administración de medicamentos como sedantes, inhibidores de la secreción gástrica ${ }^{(14)}$ y el uso prolongado de antibióticos.

La mortalidad por NIH se estima que está en el rango de 30 a 70\%, esta cifra depende de la institución y el área hospitalaria donde se realiza el estudio ${ }^{(14)}$. Los factores asociados a mal pronóstico y alta mortalidad son la enfermedad de fondo descompensada, la edad avanzada, la ventilación mecánica prolongada, APACHE II (Acute Physiology And Chronic Health Evaluation II) al ingreso, shock séptico y disfunción orgánica ${ }^{(15,16)}$.

En Lima, Perú, se llevó a cabo un estudio en la UCI del Hospital Cayetano Heredia, en donde se reportó a la NIH como la infección intrahospitalaria más frecuente con una incidencia del $28.6 \%{ }^{(1)}$. En la UCI del Hospital Daniel Alcides Carrión del Callao, un estudio encontró que la intubación endotraqueal $(p=9,608)$, la ventilación mecánica $(p=11,780)$ y la aspiración de secreciones, son los factores de riesgo más fuertemente asociados a $\mathrm{NIH}^{(17)}$. Cabanillas ${ }^{(18)}$ en el Hospital Lazarte Echegaray de Trujillo, realizó un 
estudio para determinar los factores de riesgo en paciente que presentan neumonía intrahospitalaria en el servicio de medicina interna, reportando a la intubación endotraqueal $(\mathrm{OR}=10.36)$, alteración de la conciencia $(\mathrm{OR}=11.37)$, aspiración de secreciones $(\mathrm{OR}=18)$ y uso de antiácidos $(\mathrm{OR}=4.4)$ como los más fuertemente asociados.

Por ser el HNGAI un centro de referencia nacional, con personal capacitado y habiendo escasos estudios sobre NIH en áreas de hospitalización, es que se enmarca el presente trabajo dentro del esfuerzo de determinar las características epidemiológicas más relevantes de los pacientes hospitalizados que presentan un cuadro de NIH en el servicio de medicina interna. Así, el conocer la frecuencia, distribución, y los factores asociados nos permitirá poder tomar medidas para la prevención de esta patología y lograr disminuir la estancia hospitalaria y la morbimortalidad en los servicios de hospitalización.

\section{MATERIAL Y MÉTODOS}

Se realizó un estudio descriptivo, retrospectivo. La muestra estuvo conformada por todas las historias clínicas de pacientes con el diagnóstico de $\mathrm{NIH}$, que fueron egresados del área de hospitalización del servicio de medicina interna $\mathrm{N}^{\circ} 5$ del HNGAl durante el periodo de julio a diciembre del año 2015. Se incluyeron todos los pacientes mayores de 15 años que cumplieron con los criterios de diagnóstico de $\mathrm{NIH}$ según la SAT ${ }^{(4)}$, excluyéndose del estudio los pacientes con menos de 48 horas de estancia hospitalaria y reingreso luego de haber sido dado de alta. Los datos de las historias clínicas revisadas fueron ingresados en una hoja de recolección de datos, este instrumento estuvo estructurado en dos partes. En la primera parte se reportaron los datos generales del paciente (nombre, edad, sexo), número de cama, número de historia clínica y fecha de hospitalización. La segunda parte, estuvo compuesta por preguntas en un formato con respuestas dicotómicas relacionadas a $\mathrm{NIH}$ (radiografía de tórax con infiltrado radiológico nuevo, leucocitosis o leucopenia, secreciones bronquiales purulentas, fiebre $>38^{\circ} \mathrm{C}$, agente etiológico y estancia hospitalaria $>48$ horas), y los factores intrínsecos (diabetes mellitus, enfermedad pulmonar obstructiva crónica, insuficiencia renal crónica, diálisis, enfermedad neoplásica, enfermedad cerebrovascular, enfermedad neuromuscular), y los factores extrínsecos (intubación endotraqueal, ventilación mecánica, uso de sonda nasogástrica, aspiración de secreciones, uso de inhibidores de la secreción, gástrica, traqueostomía, profilaxis de úlceras de stress). La validez del instrumento se realizó mediante un juicio de expertos, obteniéndose un índice de Kappa de 0.77. Para la confiabilidad del instrumento se realizó una prueba piloto y se obtuvo un valor en la fórmula 20 de Kuder-Richardson de 0.77 .

El análisis estadístico de los resultados se realizó mediante el paquete estadístico SPSS versión $22.0 \mathrm{y}$ los datos obtenidos fueron organizados y presentados en tablas simples de frecuencia y porcentaje.

\section{RESULTADOS}

Durante el periodo de estudio fueron egresados 1098 pacientes del servicio de medicina interna $\mathrm{N}^{\circ} 5$, de los cuales ingresaron al estudio 26 pacientes con el diagnóstico de NIH. La incidencia fue de $2.37 \%$ y la mortalidad de $73.07 \%$, con 19 pacientes fallecidos. Se encontró 17 pacientes del sexo masculino $65.38 \%$ y 9 pacientes $34.62 \%$ del sexo femenino. La edad promedio fue de $58.69 \pm 12.49$ años. La mayor proporción de casos estuvo en los pacientes mayores de 80 años, que representaron el $42.31 \%$ (11 pacientes) (Tabla 1).

Tabla 1. Distribución según edad y sexo de los pacientes con neumonía intrahospitalaria

\begin{tabular}{|c|c|c|c|c|c|c|}
\hline \multirow{2}{*}{ Grupo de edades (años) } & \multicolumn{2}{|c|}{ Masculino } & \multicolumn{2}{c|}{ Femenino } & \multicolumn{3}{|c|}{ Total } \\
\hline $40-49$ & $\mathrm{n}$ & $\%$ & $\mathrm{n}$ & $\%$ & $\mathrm{n}$ & 7.69 \\
\hline $50-59$ & 1 & 3.85 & 1 & 3.85 & 2 & 3.85 \\
\hline $60-69$ & 0 & 0.00 & 1 & 3.85 & 1 & 19.23 \\
\hline $70-79$ & 5 & 19.23 & 0 & 0.00 & 5 & 26.92 \\
\hline 80 a más & 4 & 15.38 & 3 & 11.54 & 7 & 42.31 \\
\hline Total & 7 & 26.92 & 4 & 15.38 & 11 & 100.00 \\
\hline
\end{tabular}


Respecto a la estancia hospitalaria, esta fue un promedio de 62.53 días. Se encontró que 12 pacientes $46.15 \%$ estuvieron más de 60 días en hospitalización (Tabla 2).

Tabla 2. Distribución según días de estancia hospitalaria de los pacientes con neumonía intrahospitalaria

\begin{tabular}{|c|c|c|}
\hline Estancia hospitalaria (dias) & $\mathrm{n}$ & $\%$ \\
\hline $10-19$ & 9 & 34.61 \\
\hline $20-29$ & 0 & 0.00 \\
\hline $30-39$ & 3 & 11.54 \\
\hline $40-49$ & 1 & 3.85 \\
\hline $50-59$ & 1 & 3.85 \\
\hline 60 a más & 12 & 46.15 \\
\hline Total & 26 & 100.00 \\
\hline
\end{tabular}

Tabla 3. Factores asociados a los pacientes con neumonía intrahospitalaria

\begin{tabular}{|l|c|c|}
\hline \multicolumn{1}{|c|}{ Factores asociados } & n & $\%$ \\
\hline Enfermedad cerebrovascular & 7 & 26.92 \\
\hline Enfermedad neoplásica & 6 & 23.07 \\
\hline Diabetes mellitus & 5 & 19.23 \\
\hline Insuficiencia renal crónica/diálisis & 5 & 19.23 \\
\hline EPOC & 4 & 15.38 \\
\hline Enfermedad neuromuscular & 2 & 7.69 \\
\hline Uso de sonda nasogástrica & 22 & 84.61 \\
\hline Profilaxis de úlceras de stress & 22 & 84.61 \\
\hline Aspiración de secreciones & 16 & 61.53 \\
\hline Intubación endotraqueal & 14 & 53.84 \\
\hline Ventilación mecánica & 13 & 50.00 \\
\hline Traqueostomía & 6 & 23.07 \\
\hline
\end{tabular}

La patología más frecuentemente asociada a NIH fue la enfermedad cerebrovascular con 7 pacientes $26.92 \%$, seguido de la enfermedad neoplásica con 6 pacientes $23.07 \%$. La diabetes mellitus se presentó en 5 pacientes $19.23 \%$ y la insuficiencia renal crónica en 5 pacientes $19.23 \%$. Los factores extrínsecos más frecuentemente encontrados fueron la presencia de profilaxis de úlceras de stress con 22 pacientes $84.61 \%$ y la presencia de sonda nasogástrica con 22 pacientes 84.61\%. Se encontró 16 pacientes $61.53 \%$ a quienes se les realizó aspiración de secreciones bronquiales, 14 pacientes $53.84 \%$ con intubación endotraqueal, 13 pacientes 50\% ingresaron a ventilación mecánica y 6 pacientes $23.07 \%$ fueron sometidos a traqueostomía (Tabla 3). 
Características epidemiológicas de la neumonía intrahospitalaria en un servicio

de medicina interna del Hospital Guillermo Almenara Irigoyen de Lima, Perú

Tabla 4. Microorganismo aislado en pacientes con neumonía intrahospitalaria

\begin{tabular}{|l|c|c|}
\hline Microorganismo & $\mathrm{n}$ & $\%$ \\
\hline Acinetobacter baumannii & 4 & 15.38 \\
\hline Pseudomona aeruginosa & 3 & 11.53 \\
\hline Staphilococcus aureus & 1 & 3.84 \\
\hline Enterobacter aerogenes & 1 & 3.84 \\
\hline No aislado & 17 & 65.38 \\
\hline Total & 26 & 100.00 \\
\hline
\end{tabular}

Los microorganismo aislados en el presente estudio fueron Acinetobacter baumannii con 15.38\%, Pseudomona aeruginosa con $11.53 \%$, Staphilococcus

\section{DISCUSIÓN}

La reducción en la incidencia de las infecciones intrahospitalarias se ha convertido en un desafío al que se enfrenta la comunidad médica científica, ya que aún se puede distinguir en las diferentes áreas hospitalarias de alta y menor complejidad que la NIH aparece como la infección más frecuente. El presente trabajo se realizó en los últimos seis meses del año, meses de otoño e invierno con mayor proporción de infecciones respiratorias. Durante el periodo que comprendió el estudio se reportó que la incidencia de NIH fue de 2.37\%; valor similar al publicado por Cabanillas ${ }^{(18)}$ que reportó $2.25 \%$ en un estudio que realizó en el servicio de medicina interna del Hospital "Víctor Lazarte Echegaray" de Trujillo, en un periodo de tiempo, área hospitalaria y época del año similares a nuestro estudio. La NIH es la infección más frecuente en $\mathrm{UCl}$ y la mayoría de estudios han sido llevados a cabo en este ámbito, de allí que la incidencia que reportan diversos trabajos es mucho mayor al de nuestro estudio, como Otiniano y Gómez ${ }^{(17)}$ que reportaron una incidencia del $11 \%$ y Chincha y col (1) de $28.6 \%$, en ambos estudios los pacientes estuvieron intubados y en ventilación mecánica, lo que favoreció la mayor cantidad de casos de NIH.

En nuestro trabajo se encontró que el $65.38 \%$ de pacientes pertenecieron al género masculino. Esta cifra es similar al encontrado por Sopena y col..$^{(19)} \mathrm{con}$ un $72.3 \%$ y el de Magret y col. $^{(7)}$ con un $71.4 \%$ de pacientes varones.

El promedio de edad de los pacientes del estudio fue de 58.69 años, con el $42.31 \%$ en el grupo de edad de mayores de 80 años. Resultados similares a los aureus con 3.84\%, Enterobacter aerogenes con 3.84\%. No se llegó a aislar ningún agente patógeno en el $65.38 \%$ de pacientes (Tabla 4 ).

encontrados por Sopena y col. ${ }^{(19)}$ que reportaron una edad promedio de 70 años y Cabanillas ${ }^{(18)}$ de 78.1 años. Estas cifras nos indican que la NIH es una infección con tendencia a incrementar su frecuencia conforme aumenta la edad de los pacientes. El envejecimiento provoca una serie de cambios en la fisiología respiratoria del anciano, además de la disminución del sistema inmune y las comorbilidades del paciente. Estos factores se combinaron para que la mayor proporción de casos de NIH se presente en la población adulta y anciana.

Respecto a la estancia hospitalaria, el promedio fue de 62.53 días. Contrario a lo descrito por otros autores que reportan una estancia de 14.8 días $^{(17)}$, y 12 días ${ }^{(20)}$ en $\mathrm{UCl}$. Nuestro servicio de medicina alberga pacientes con múltiples comorbilidades, lo que deriva en una elevada estancia hospitalaria.

En el presente estudio, la enfermedad cerebrovascular que derivó en trastorno de conciencia, se presentó en $26.92 \%$. Los diferentes estudios reportan cifras mayores como Otiniano y Gómez ${ }^{(17)} 72.72 \%$ y Cabanillas ${ }^{(18)} 73.52 \%$. Dichos estudios reportaron el mecanismo que llevó a la microaspiración, como es la alteración del estado de conciencia, mientras que en nuestro estudio se reportó la comorbilidad asociada que llevó a la alteración del nivel de conciencia como la enfermedad cerebrovascular, lo cual explica la diferencia en los resultados. La alteración del estado de conciencia es un factor de riesgo conocido para desarrollar NIH, pues facilita la microaspiración de secreciones traqueobronquiales.

Se reportó $19.23 \%$ de pacientes con diabetes mellitus. En el estudio de Otiniano y Gómez ${ }^{(17)}$ se reportó 
$3.03 \%$. La diabetes mellitus es una enfermedad metabólica crónica caracterizada por disminuir el sistema inmunológico, sin embargo esta proporción escasa de pacientes nos indica, como lo reportan diferentes autores, que esta patología metabólica no tiene trascendencia como factor de riesgo en el desarrollo de la $\mathrm{NIH}^{(8,9,10)}$.

Se encontró el $84.61 \%$ de pacientes con profilaxis de úlceras de stress con agentes inhibidores de la secreción gástrica. Resultados semejantes reportaron Herzig y col. ${ }^{(14)} 80 \%$, Cabanillas ${ }^{(18)}(82.35 \%)$. Estos estudios demuestran que es una indicación muy frecuente que los pacientes hospitalizados en los servicios de medicina y más aún en $\mathrm{UCl}$ reciban inhibidores de la secreción gástrica, lo que explica el porcentaje elevado de pacientes con estos medicamentos.

El $84.61 \%$ de pacientes fueron usuarios de sonda nasogástrica. Valores similares a los reportados por otros autores como Cabanillas ${ }^{(18)} 85.35 \%$ y de Otiniano y Gómez ${ }^{(17)} 96.96 \%$. En nuestro servicio la indicación para colocación de sonda nasogástrica en pacientes adultos y ancianos para asegurar la alimentación es una práctica común que refleja la alta proporción de pacientes con esta condición.

El porcentaje pacientes con intubación endotraqueal fue de $53.84 \%$ y los que ingresaron a ventilación mecánica de $50 \%$. Cabanillas ${ }^{(18)}$ encontró $29.41 \%$ de pacientes con intubación endotraqueal en un área de medicina interna. Los estudios llevados a cabo en $\mathrm{UCl}$ reportan el $100 \%$ de pacientes intubados y en ventilación mecánica ${ }^{(16,17)}$. Esta diferencia está relacionada con el área hospitalaria donde se realizó el estudio, con valores menores en los servicios de medicina interna donde no todos los pacientes llegan a ser intubados ni tampoco ingresan a ventilación mecánica.

En relación a los microorganismos aislados, los más frecuentes fueron Acinetobacter baumannii 15.38\% y Pseudomona aeruginosa $11.53 \%$. Similares resultados fueron reportados por Otiniano y Gómez ${ }^{(17)}$, quienes aislaron Pseudomona aeruginosa con $26.47 \%$ como el microorganismo más frecuente y Weyland y col. ${ }^{(6)}$ que aislaron como gérmenes más frecuentes Acinetobacter baumannii $37.9 \%$ y Pseudomona aeruginosa $20.9 \%$. Estos resultados reafirman que los gérmenes gram negativos son aislados en mayor proporción en los pacientes con $\mathrm{NIH}$. Se destaca, sin embargo, en nuestro estudio que en el $65.38 \%$ de pacientes no se logró aislar el agente etiológico.
Montalvo y col. ${ }^{(15)}$ no identifican al agente causal en el $26.75 \%$ de los casos. La escasa proporción de gérmenes aislados en nuestro estudio se puede explicar debido a que en el momento de la obtención de la muestra muchos pacientes estaban bajo tratamiento antibiótico; así mismo, en otros pacientes no se pudo realizar la toma de la muestra por problemas técnicos operativos necesarios para esta finalidad.

La mortalidad en nuestro estudio fue de $73.07 \%$, mientras que en el estudio de Uvizl y col. ${ }^{(21)}$ esta fue de $35 \%$. La SAT ${ }^{(4)}$ reportó un rango de $30 \%$ a $70 \%$ en la tasa de mortalidad por $\mathrm{NIH}$. Este rango tan amplio de mortalidad está en relación a la disparidad de criterios diagnósticos, al área hospitalaria y la latitud donde se realizó el estudio. Guzmán-Herrador y col. ${ }^{(22)}$ reportaron que el desarrollo de $\mathrm{NIH}$ en los pacientes en $\mathrm{UCl}$, fue un factor independiente para alta mortalidad en dicho ámbito hospitalario. Magret y col. ${ }^{(7)}$ en su estudio sobre mortalidad concluyeron que la estancia hospitalaria prolongada y los gérmenes gram negativos aislados son un factor de riesgo independiente para mortalidad. Estas dos circunstancias se presentaron en nuestro estudio con la mayoría de gérmenes aislados y una estancia hospitalaria prolongada, lo que derivó en una alta tasa de mortalidad en el presente trabajo.

El presente trabajo tiene las limitaciones de un estudio retrospectivo con información obtenida de las historias clínicas de pacientes con una patología respiratoria e infecciosa que a pesar de ser muy frecuente los servicios de medicina, se obtuvo en los últimos seis meses del año un tamaño de muestra pequeño, que se reflejó en la incidencia de la enfermedad. No obstante, ser un estudio descriptivo realizado en un corto periodo de tiempo y en época invernal, podemos afirmar que los pacientes que presentan $\mathrm{NIH}$ en el servicio de medicina interna tienen una alta mortalidad asociada.

El no aislarse microorganismos en un alto porcentaje de casos pudo evitarse con un estudio de tipo prospectivo. Se requiere de estudios posteriores de tipo analítico para determinar la asociación entre los factores asociados tales como la edad, sexo masculino, el uso de sonda nasogástrica, la profilaxis de úlceras de stress y estancia hospitalaria prolongada en el desarrollo de $\mathrm{NIH}$.

En conclusión, los pacientes con NIH fueron en su mayoría adultos mayores con enfermedades crónicas y procedimientos invasivos que condicionaron una estancia prolongada. 
Características epidemiológicas de la neumonía intrahospitalaria en un servicio de medicina interna del Hospital Guillermo Almenara Irigoyen de Lima, Perú

Los principales factores asociados a NIH fueron la enfermedad cerebrovascular, enfermedad neoplásica, y el uso de sonda nasogástrica y profilaxis de úlceras de stress.

La NIH, en el servicio de medicina interna, tiene una alta mortalidad; no obstante el corto periodo de tiempo del estudio.

\section{Recomendaciones}

1. El presente estudio servirá de referencia para posteriores trabajos sobre $\mathrm{NIH}$, sugiriéndose un diseño analítico y ampliar el periodo de tiempo para un mejor análisis del ámbito donde se realice el estudio.

2. Dada la mortalidad de NIH en el servicio de medicina interna es necesario realizar un diagnóstico precoz y tratamiento oportuno de esta afección.

Agradecimiento: A Hugo Gutiérrez Crespo, maestro de la Universidad Nacional Mayor de San Marcos y al staff médico del Servicio de Medicina Interna $\mathrm{N}^{\circ} 5$ del Hospital Guillermo Almenara Irigoyen por su apoyo y motivación para terminar el trabajo.

\section{REFERENCIAS BIBLIOGRÁFICAS}

1. Chincha O, Cornelio E, Valverde V, Acevedo M. Infecciones Intrahospitalarias asociadas a dispositivos invasivos en Unidad de Cuidados Intensivos de un Hospital Nacional de Lima, Perú. Rev Perú Med Exp Salud Pública. 2013;30(4):616-20.

2. Diaz E, Martin-Loeches I, Valles J. Neumonía Nosocomial. Enferm Infecc Microbiol Clin. 2013;31(10):692-98

3. Blanquer J, Aspa J, Anzueto A, Ferrer M, Gallego M, Rajas O, et al. Normativa SEPAR: Neumonía Nosocomial. Arch Bronconeumol. 2011;47(10):510-20.

4. American Thoracic Society. Guidelines for the Management of Adults with Hospital-acquire, Ventilator-associated, and Healthcare-associated Pneumonia. Am J Respir Crit Care Med. 2005;171:388-416.

5. Dalhoff K, Ewing S, Guideline Development Group, Abele-Horn $M$, Andreas S, Bauer T, et al. Adult Patients With Nosocomial Pneumonia. Dtsch Arztebl Int. 2013;110(38):634-40.

6. Weyland B, Perazzi B, García S, Rodriguez C, Vay C, Famiglietti A. Etiología bacteriana de la neumonía nosocomial y resistencia a los antimicrobianos en pacientes con y sin tratamiento antimicrobiano previo. Rev Argent Microbiol. 2011;41(1):18-23.

7. Magret M, Lisboa T, Martin-Loeches I, Mañez R, Nauwynck M, Wrigge $\mathrm{H}$. et al. Bacteriemia is an independent risk factor for mortality in nosocomial pneumonia: a prospective and observational multicenter study. Crit Care. 2011;15(1).

8. Tsakiridou E, Makris D, Chatzipantazi V, Vlachos O, Xidopoulos G, Charalampidou $\mathrm{O}$ et at. Diabetes and Hemoglobin A1c as Risk Factors for Nosocomial Infections in Critically III Patients. Crit Care Res Pract 2013;2013:279479.

9. Graham B, Keniston A, Gatic O, Trillo C, Medvedev S, Douglas I. Diabetes mellitus does not adversely affect outcomes from a critical illness. Crit Care Med. 2010;38(1):16-18.
10. Siegelaar S, Hickmann M, Hoekstra JB, Holleman F, DeVries J. The effect of diabetes on mortality in critically ill patients: a systematic review and meta-analysis. Crit Care. 2011;15(5)R205.doi: 10.1189/cc10440.

11. Divani AA, Hevesi M, Pulivarthi S, Luo X, Souslian F, Suarez JI, et al. Predictor of Nosocomial Pneumonia in Intracerebral Hemorrhage Patients: A Multi-center Observational Study. Neurocritic Care. 2015; 22(2): 234-42.

12. Friedant, A, Gouse B, Boehme A, Siegler J, Albright K, Monlezun $D$, et al. A simple prediction score for developing a hospitalacquired infection after acute ischemic stroke. J Stroke Cerebrovasc Dis. 2015;24(3):680-6.

13. Nordin N, Kamaruzzamam SB, Chin AV, Poi PJ, Tan MP. A descriptive study of nasogastric tube feeding among geriatric inpatient in Malaysia: utilization, complications, and caregiver opinions. J Nutr Gerontol Geriatric. 2015;34(1):34-9.

14. Herzig S, Doughty C, Lahoti S, Marchina S, Sanan N, Feng W et al. Acid-suppresive medication use in acute stroke and hospitaladquired pneumonia. Ann Neurol. 2014;76(5):712-8.

15. Montalvo R, Alvarezcano J, Huaroto L, López J, Lam C, Mucha R, et al. Factores asociados a mortalidad por neumonía nosocomial en un hospital público del Perú. Rev Perú Epidemiol. 2013;17(2):1-6.

16. Kuo-Tung H, Chia Cheng T, Wen-Feng F, Meng-Chih, L. An early predictor of the outcome of patients with ventilator-associated pneumonia. Chang Gung Med J. 2010;33(3):274-82.

17. Otiniano A, Gómez M. Factores de riesgo asociados a neumonía intrahospitalaria en pacientes de la unidad de cuidados intensivos. Rev Soc Peruana Med Interna. 2011;24(3):121-127.

18. Cabanillas E. Factores de riesgo asociados a neumonía intrahospitalaria en pacientes del Servicio de Medicina del Hospital Víctor Lazarte Echegaray. (Tesis Título de Especialista).Trujillo: Biblioteca Digital Oficina de sistemas e informática. Universidad Nacional de Trujillo. 2009.

19. Sopena N, Heras E, Casas I, Bechini J, Guasch I, Pedro- Botet M et al. Risk factors for hospital-acquired pneumonia outside the intensive care unit: A case control study. Am J Infect Control. 2014 Jan;42(1):38-42.

20. Vanhems P. Nosocomial pulmonary infection by antimicrobialresistant of patients hospitalized in intensive care units: risk factors and survival. J Hosp Infect. 2000;45:98-106.

21. Uvizl R, Hanulik V, Husickova V, Sedlakova MH, Adamus M, Kolar, M. Hospital-adquired pneumonia in UCl patients. Biomed Pap Med Fac Univ Palacky Olomovc Czech Repub.2011;155(4):373-8.

22. Guzman-Herrador B, Diaz C, Allan M, Fernandez-Crehuet N. Underlying illness severity and outcome of nosocomial pneumonia: prospective cohort study in intensive care unit. J Hosp Infect. 2014;86(1):53-6.

\section{Fuentes de financiamiento}

Este artículo ha sido financiado por los autores.

\section{Conflictos de interés}

Los autores declaran no tener ningún conflicto de interés.

\section{Correspondencia}

Cesar Jacinto León Chahua

Dirección: Av. Universitaria Sur 1951. Pueblo Libre, Lima. Perú.

Teléfono: 01-4618478

Correo electrónico: cesarleonch@hotmail.com 